\title{
A Differential Evolution Algorithm to Semivectorial Bilevel Problems
}

\author{
Maria João Alves ${ }^{1,3}$, Carlos Henggeler Antunes ${ }^{2,3}$ \\ ${ }^{1}$ CEBER - Faculty of Economics, University of Coimbra, Portugal \\ ${ }^{2}$ DEEC, University of Coimbra, Polo 2, Coimbra, Portugal \\ ${ }^{3}$ INESC Coimbra, Coimbra, Portugal \\ mjalves@fe.uc.pt; ch@deec.uc.pt
}

\begin{abstract}
Semivectorial bilevel problems (SVBLP) deal with the optimization of a single function at the upper level and multiple objective functions at the lower level of hierarchical decisions. Therefore, a set of nondominated solutions to the lower level decision maker (the follower) exists and should be exploited for each setting of decision variables controlled by the upper level decision maker (the leader). This paper presents a new algorithmic approach based on differential evolution to compute a set of four extreme solutions to the SVBLP. These solutions capture not just the optimistic vs. pessimistic leader's attitude but also possible follower's reactions more or less favorable to the leader within the lower level nondominated solution set. The differential evolution approach is compared with a particle swarm optimization algorithm. In this experimental comparison we draw attention to pitfalls associated with the interpretation of results and assessment of the performance of algorithms in SVBLP.
\end{abstract}

Keywords: semivectorial bilevel problems; differential evolution; particle swarm optimization; optimistic/pessimistic frontiers; optimistic/deceiving solutions; pessimistic/rewarding solutions

\section{Introduction}

A semivectorial bilevel problem (SVBLP) is an optimization problem with a single objective function at the upper (leader's) level and multiple objective functions at the lower (follower's) level of hierarchical non-cooperative decisions. Hence, a multiobjective (MO) optimization problem contributes to define the feasible region to the leader's problem, in the sense that a lower level nondominated region exists for each setting of upper level variables. Thus, when solving his/her optimization problem, the leader must anticipate the follower's choice of a nondominated solution embodying a trade-off between the lower level multiple objectives. The follower's reaction may strongly affect the leader's optimal solution, depending on the follower's preference structure vis-àvis the nondominated region established by the instantiation of the leader's decision variables. Therefore, it is useful for the leader to have an overview of possible optimal solutions resulting from different attitudes (optimistic or pessimistic) in face of his/her 
expectation of the more or less favorable follower's choice. In addition to the intrinsic theoretical and computational difficulty in computing solutions to the SVBLP, the leader does not have a-priori information about the nondominated solution the follower will choose according to his/her (unknown) preferences.

In this setting, this paper presents an algorithmic approach intertwining single and MO versions of Differential Evolution (DE) for the upper level and lower level problems, which is aimed at computing a set of extreme solutions to the SVBLP. These extreme solutions are: the optimistic solution offering the leader the best objective function value when the follower's decision for each setting of upper level variables is the best for the leader; the deceiving solution when the leader adopts an optimistic approach but the follower's reaction is the worst for the leader; the pessimistic solution offering the best objective function value for the leader when the follower's decision for each setting of upper level variables is the worst for the leader; and the rewarding solution when the leader adopts a pessimistic approach but the follower's reaction is the most favorable to the leader.

The algorithmic approach introduces new concepts of optimistic and pessimistic frontiers and adapts DE mechanisms to combine the search at both levels with the population split between orientations to each frontier. This approach is compared with a Particle Swarm Optimization (PSO) algorithm we have previously developed [1], which has been extended herein to compute these four extreme solutions. The algorithms are tested on a set of benchmark problems for multiobjective bilevel (MOBL) optimization (considering only one of the objective functions in the upper level). We were able to determine analytically the exact solutions to these problems, which enable to assess the quality of the solutions obtained by the algorithms. A thorough analysis of the computational results allowed us to unveil pitfalls associated with the interpretation of results and assessment of the algorithm performance in SVBL and MOBL optimization. This paper also aims at drawing attention to these pitfalls.

In section 2, the SVBLP is presented and the definitions of the extreme (optimistic, deceiving, pessimistic and rewarding) solutions are introduced. Algorithmic approaches to deal with the SVBLP are also briefly reviewed in this section. The concepts of optimistic and pessimistic frontiers are presented and illustrated in section 3. In section 4, the Semivectorial Bilevel Differential Evolution (SVBLDE) algorithm is proposed. Computational results are presented and discussed in section 5. Concluding remarks are presented in section 6 .

\section{The SVBLP: Optimistic vs. Pessimistic Approaches}

The SVBLP is a bilevel optimization problem with a single objective function at the upper level $F(x, y)$ and multiple objective functions $f_{k}(x, y), k=1, \ldots, m$ at the lower level.

$$
\begin{array}{ll}
\text { 'min } & F(x, y) \\
\text { s.t. } & G(x, y) \leq 0 \\
& y \in \arg \min _{y \in Y}\left\{\left(f_{1}(x, y), \ldots, f_{m}(x, y)\right): g(x, y) \leq 0\right\}
\end{array}
$$


with $X \subseteq \mathfrak{R}^{n_{1}}$ and $Y \subseteq \mathfrak{R}^{n_{2}}$, which impose bounds (box constraints) on the upper level variables $x$ (which are controlled by the leader) and on the lower level variables $y$ (which are controlled by the follower), respectively. $G(x, y) \leq 0$ and $g(x, y) \leq 0$ are general constraints, respectively in the upper and the lower level problems.

Since the decision process is sequential and the leader decides first, $x$ assumes a constant vector in the optimization of $f_{k}(x, y), k=1, \ldots, m$. For each $x \in X$ there is a set of efficient (Pareto optimal or nondominated) solutions to the lower level problem represented by $\Psi_{E f}(x)$. Let $Y(x)=\{y \in Y: g(x, y) \leq 0\}$.

Thus, $\Psi_{E f}(x)=\left\{y \in Y:\left(\right.\right.$ there is no $\left.\left.y^{\prime} \in Y(x) \mid f\left(x, y^{\prime}\right) \prec f(x, y)\right)\right\}$ where $\prec$ denotes the dominance relation, i.e., $f\left(x, y^{\prime}\right) \prec f(x, y)$ iff $f_{j}\left(x, y^{\prime}\right) \leq f_{j}(x, y)$ for all $j=1, \ldots, m$, and $f_{j}\left(x, y^{\prime}\right)<f_{j}(x, y)$ for at least one $j$.

Since there is not, in general, a single efficient solution to the lower level problem for each $x$, problem (1) is ambiguous. This is the reason for the quotation marks in the upper level objective function. Two main approaches have been suggested in the literature to address the problem - the optimistic and the pessimistic approaches - leading to two reformulations of (1). As in the single objective bilevel problem with non-unique optimal solutions to the lower level problem, the optimistic formulation of the SVBLP is much simpler to tackle and has therefore been the most investigated.

The optimistic approach assumes that the leader is able to influence the choice of the follower. Thus, the upper level optimization can be taken with respect to $x$ and $y$ to determine the optimal optimistic solution. This means that, for a given upper level decision $x$, the lower level decision $y$ is the one that presents the minimum $F(x, y)$ among the efficient solutions to the lower level problem for that $x$, which also satisfy upper level constraints (if there are upper level constraints coupled with lower level variables, i.e. $G(x, y) \leq 0)$. The optimal optimistic solution will be called just optimistic solution and is defined as follows:

- The optimistic solution, $\left(x^{\mathrm{o}}, y^{\mathrm{o}}\right)$, is given by

$$
\min _{x \in X, y \in Y}\left\{F(x, y): y \in \Psi_{E f}(x), G(x, y) \leq 0\right\}
$$

In the pessimistic approach the leader prepares for the worst case. The leader chooses the $x$ that leads to a feasible solution with minimum $F$ in view of the follower's decisions $y$ worst for the leader. The optimal pessimistic solution will be called just pessimistic solution and is defined as follows:

- the pessimistic solution, $\left(x^{\mathrm{p}}, y^{\mathrm{p}}\right)$, is given by

$$
\min _{x \in X}\left\{\max _{y \in Y}\left\{F(x, y): y \in \Psi_{E f}(x)\right\}: G(x, y) \leq 0\right\}
$$

A failed optimistic approach leads to the deceiving solution. This means that the leader chooses $x$ according to the optimistic approach but the follower does not react accordingly and takes the decision with worst value for the leader's objective function. Thus, given the optimistic upper level decision $x^{\mathrm{o}}$, 
- the deceiving solution is $\left(x^{\mathrm{d}}, y^{\mathrm{d}}\right)=\left(x^{\mathrm{o}}, y^{\mathrm{d}}\right)$ where $y^{\mathrm{d}}$ is given by $\max _{y \in Y}\left\{F\left(x^{\mathrm{o}}, y\right): y \in \Psi_{E f}\left(x^{\mathrm{o}}\right)\right\}$.

According to the above definition, the deceiving solution may be infeasible to the leader, i.e. infeasible for the SVBLP. Knowing whether the deceiving follower's reaction is feasible or infeasible to the upper level problem is also a useful information to the leader.

A successful pessimistic approach leads to the rewarding solution. Thus, given the pessimistic upper level decision $x^{\mathrm{p}}$, the rewarding solution can be defined as the feasible $\left(x^{\mathrm{r}}, y^{\mathrm{r}}\right)=\left(x^{\mathrm{p}}, y^{\mathrm{r}}\right)$ such that $y^{\mathrm{r}}$ is given by

$$
\min _{y \in Y}\left\{F\left(x^{\mathrm{p}}, y\right): y \in \Psi_{E f}\left(x^{\mathrm{p}}\right), G\left(x^{\mathrm{p}}, y\right) \leq 0\right\}
$$

Bonnel [2] and Bonnel and Morgan [3] firstly addressed the SVBLP by providing necessary optimality conditions [2] and a penalty function method [3] for determining the optimistic solution. Other methods based on penalty functions to compute the optimistic solution were developed by Ankhili and Mansouri [4], Zheng and Wan [5] and Ren and Wang [6] for the SVBLP with a MO linear problem in the lower level. Calvete and Galé [7] focused on the same problem and proposed an exact method and a genetic algorithm, considering the optimistic approach. Liu et al. [8] developed necessary optimality conditions for the pessimistic solution and Lv and Chen [9] proposed a discretization iterative algorithm to compute the pessimistic solution to a SVBLP without upper level variables in the lower level constraints. Alves et al. [1] firstly introduced the concept of deceiving solution and proposed an algorithm based on PSO to approximate the optimistic, pessimistic and deceiving solutions to the SVBLP. The rewarding solution was introduced in [10], where illustrative examples of these four types of extreme solutions were presented. In the present paper we propose a new algorithm based on DE to compute these four extreme solutions and extend the algorithm in [1] to compute also the rewarding solution.

\section{Optimistic and Pessimistic Frontiers}

Let us now define two new concepts to be used in the algorithm proposed in the next section, which are the Optimistic and the Pessimistic frontiers.

The Optimistic frontier $(O)$ consists of the feasible solutions $\left(x, y^{\prime}\right)$, such that $y^{\prime}$ is the follower's efficient solution $y^{\prime} \in \Psi_{E f}(x), G\left(x, y^{\prime}\right) \leq 0$, that provides the minimum (best) $F$ for that $x \in X$ :

$$
O=\left\{\left(x, y^{\prime}\right): x \in X, y^{\prime} \in \underset{y \in Y}{\arg \min }\left\{F(x, y): y \in \Psi_{E f}(x), G(x, y) \leq 0\right\}\right\}
$$

The optimistic solution $\left(x^{\mathrm{o}}, y^{0}\right)$ to the SVBLP is the solution $\left(x, y^{\prime}\right) \in O$ with minimum $F$.

The Pessimistic frontier $(P)$ consists of the solutions $\left(x, y^{\prime \prime}\right)$ such that $y^{\prime \prime}$ is the follower's efficient solution $y^{\prime \prime} \in \Psi_{E f}(x)$ that provides the maximum (worst) $F$ for that $x \in X$ : 


$$
P=\left\{\left(x, y^{\prime \prime}\right): x \in X, y^{\prime \prime} \in \underset{y \in Y}{\arg \max }\left\{F(x, y): y \in \Psi_{E f}(x)\right\}\right\}
$$

The pessimistic solution $\left(x^{\mathrm{p}}, y^{\mathrm{p}}\right)$ to the $\operatorname{SVBLP}$ is the feasible solution $\left(x, y^{\prime \prime}\right) \in P$, $G\left(x, y^{\prime \prime}\right) \leq 0$, with minimum $F$.

The deceiving solution $\left(x^{\mathrm{d}}, y^{\mathrm{d}}\right)$ is the solution in $P$ with $x^{\mathrm{d}}=x^{\mathrm{o}}$.

The rewarding solution $\left(x^{\mathrm{r}}, y^{\mathrm{r}}\right)$ is the solution in $O$ with $x^{\mathrm{r}}=x^{\mathrm{p}}$.

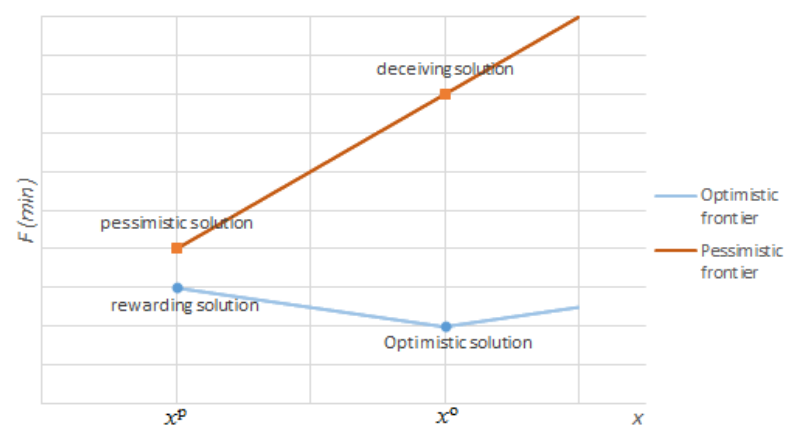

Fig. 1. $F$ values in the Optimistic and Pessimistic efficient frontiers of a SVBL linear problem with one upper level variable $(x)$ and two objective functions at the lower level.

In the example in Fig. 1, there is a significant difference between the optimistic and the deceiving solutions for the leader's objective function. Therefore, if the leader opts for an optimistic approach he/she takes a high risk, since the deceiving solution is very bad. Conversely, there is a small difference between the pessimistic $F$ and the rewarding one, being the $F$ value in the rewarding solution close to the optimistic $F$.

Since the deceiving solution is obtained from the Pessimistic frontier using an optimistic approach and the rewarding solution is obtained from the Optimistic frontier using a pessimistic approach, both frontiers should be simultaneously explored by an algorithm aimed at computing these four extreme solutions.

\section{A Differential Evolution Algorithm for the SVBLP}

In the SVBLDE algorithm proposed below, the population Pop of individuals is divided into two sub-populations $P o p^{\prime}$ and $P o p^{\prime \prime}$ which share the upper level $x$ vectors. Let $N u$ be the number of upper level individuals. Pop $=P_{o p}^{\prime} \cup P_{o p}{ }^{\prime \prime}$ where Pop' $=$ $\left\{\left(x_{1}, y_{1}^{\prime}\right),\left(x_{2}, y_{2}^{\prime}\right), \ldots,\left(x_{N u}, y_{N u}^{\prime}\right)\right\}$ and $P o p^{\prime \prime}=\left\{\left(x_{1}, y_{1}^{\prime \prime}\right),\left(x_{2}, y_{2}^{\prime \prime}\right), \ldots,\left(x_{N u}, y_{N u}^{\prime \prime}\right)\right\}$. The individuals of Pop' aim at approximating the Optimistic frontier while the individuals of Pop" aim at approximating the Pessimistic frontier. DE operations are employed to evolve the population of the upper level problem and, for each upper level vector $x$, a lower level DE algorithm (DE_LOWERLEVEL_O_P) is used to determine $\left(x, y^{\prime}\right)$ and $\left(x, y^{\prime \prime}\right)$. Below, $\varsubsetneqq$ denotes the mutation scaling factor and CR the crossover rate in the DE operations. Let 
$T u$ be the number of upper level generations. The DE upper level search is described in Algorithm 1. We have used $₹=0.7$ and $C R=0.9$.

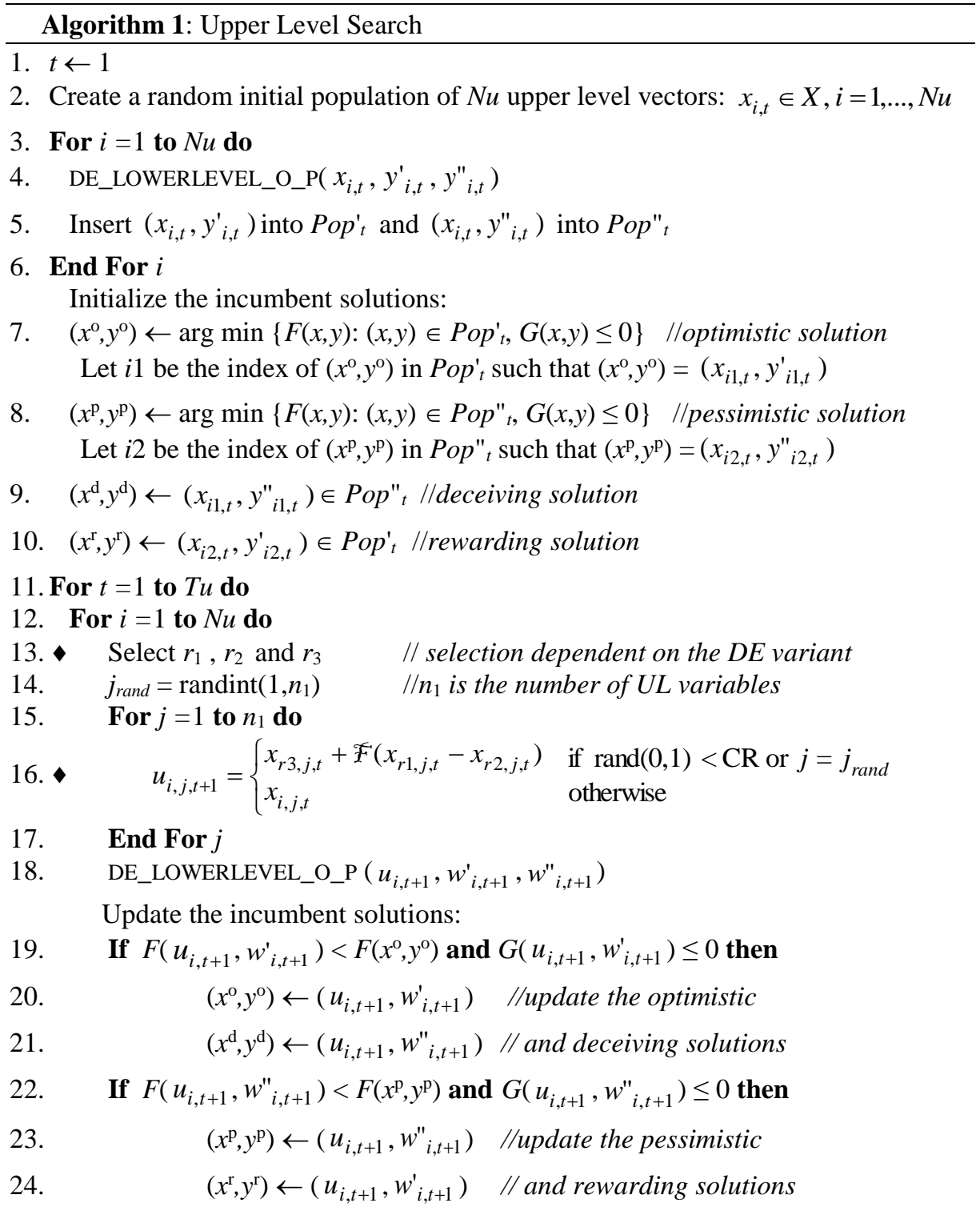

25. If ACCEPT $u_{i, t+1}$ then // criterion dependent on the DE variant

26. $\quad x_{i, t+1}=u_{i, t+1}$

27. Insert $\left(u_{i, t+1}, w_{i, t+1}^{\prime}\right)$ into Pop ${ }_{t+1}^{\prime}$ and $\left(u_{i, t+1}, w_{i, t+1}^{\prime \prime}\right)$ into Pop" ${ }_{t+1}$

28. Else 
29.

$$
x_{i, t+1}=x_{i, t}
$$

30.

$$
\text { Insert }\left(x_{i, t}, y_{i, t}^{\prime}\right) \text { into Pop }{ }_{t+1}^{\prime} \text { and }\left(x_{i, t}, y_{i, t}^{\prime \prime}\right) \text { into Pop" }{ }_{t+1}
$$

\section{End For $i$}

\section{End For $t$}

Output: $\left(x^{\mathrm{o}}, y^{\mathrm{o}}\right),\left(x^{\mathrm{d}}, y^{\mathrm{d}}\right),\left(x^{\mathrm{p}}, y^{\mathrm{p}}\right),\left(x^{\mathrm{r}}, y^{\mathrm{r}}\right)$

In Step 16, if $u_{i, j, t+1}$ does not satisfy the bounds defined by $X$, then it is projected into the closest bound.

We consider two DE variants: $D E /$ rand/l/bin (the original version, which obtained good results in the comparative study of DE variants for global optimization in [11]) and $D E / b e s t / 1 / b i n$ (the variant with highest performance in the same study). The steps marked with $\bullet$ change from one variant to the other. In Step 13, DE/rand/l/bin randomly selects indexes $r_{1} \neq r_{2} \neq r_{3}$ from $\{1, \ldots, N u\}$, while $D E /$ best $/ 1 /$ bin randomly selects indexes $r_{1} \neq r_{2}$ for $x_{r 1}$ and $x_{r 2}$ but an $x_{b e s t}$ is used in Step 16 to replace $x_{r 3}$. The $D E / b e s t / 1 / b i n$ variant divides the population into two equal parts: the first half is mainly oriented towards the optimistic solution, so $x_{\text {best }}=x^{0}$, and the second half of the population is mainly oriented towards the pessimistic solution, so $x_{\text {best }}=x^{\mathrm{p}}$. In addition, $r_{1}$ and $r_{2}$ are randomly selected from $\{1, \ldots, N u / 2\}$ for $i \leq N u / 2$ and from $\{N u / 2+1, \ldots, N u\}$ otherwise.

The criterion to decide whether $u_{i, t+1}$ is accepted or not in Step 25 (ACCEPT) also depends on the DE variant. Steps 25-30 define the population for the next generation. In $D E / \mathrm{rand} / 1 / \mathrm{bin}$, if (a) the new individual obtained for approximating the Optimistic frontier $\left(u_{i, t+1}, w_{i, t+1}^{\prime}\right)$ improves the current one in Pop't, i.e. $F\left(u_{i, t+1}, w_{i, t+1}^{\prime}\right)<F\left(x_{i, t}, y_{i, t}^{\prime}\right)$

, or $(b)$ the new individual obtained for approximating the Pessimistic frontier $\left(u_{i, t+1}, w_{i, t+1}\right)$ improves the current one in $P o p^{\prime \prime}$, i.e. $F\left(u_{i, t+1}, w_{i, t+1}^{\prime \prime}\right)<F\left(x_{i, t}, y_{i, t}^{\prime \prime}\right)$, then the new upper level individual $u_{i, t+1}$ is accepted and Steps 26-27 are performed.

Otherwise, the previous individual is kept and Steps 29-30 are performed. In $D E / b e s t / 1 / b i n$, the acceptance criterion in the first half of the population (oriented to the optimistic solution) only considers condition (a) to decide whether $u_{i, t+1}$ is accepted or not, whereas in the second half of the population only condition $(\boldsymbol{b})$ is considered.

The DE_LOWERLEVEL_O_P algorithm aims at computing two extreme efficient solutions to the lower level problem for a given $x$, one belonging to the Optimistic frontier and the other belonging to the Pessimistic frontier: $\left(x, y^{\prime}\right) \in O$ and $\left(x, y^{\prime \prime}\right) \in P$.

Let $T l$ be the number of lower level generations and $N l$ (an even number) the size of the lower level population. The algorithm attempts to converge to a population Popy of efficient solutions to the lower level problem polarized to the extreme values of the upper level objective function $F$ (the maximum and the minimum). The first $N I / 2$ individuals of Popy are oriented to converge to $y^{\prime}$ while the remaining $N l / 2$ individuals are oriented to converge to $y^{\prime \prime}$. 


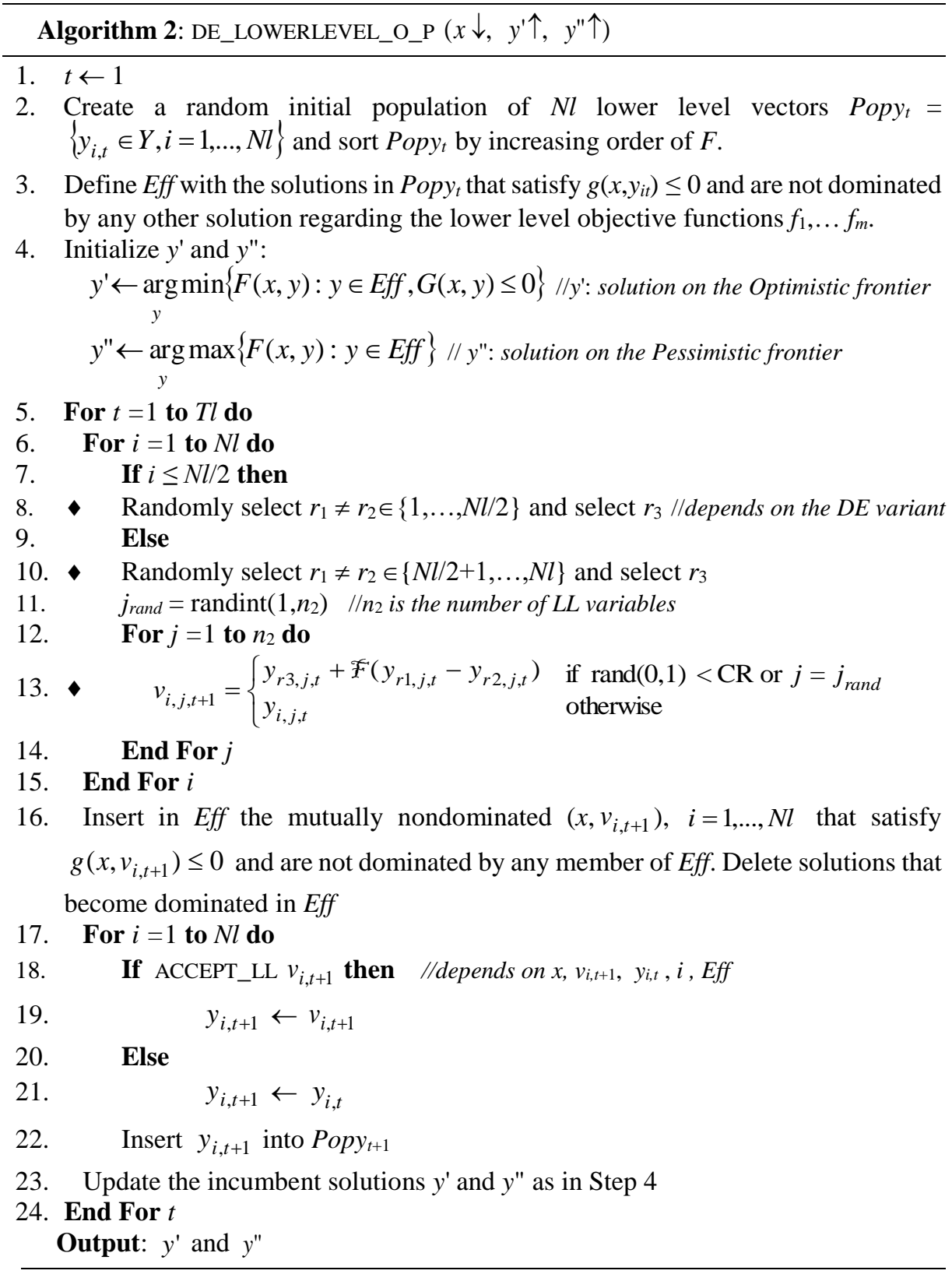

In step 2, for each new $y_{i, t} \in Y$ randomly generated, the lower level constraints $g\left(x, y_{i, t}\right) \leq 0$ are checked; if the constraints are violated then another $y_{i, t} \in Y$ is drawn. If the first and the second trials are infeasible, the solution with smaller overall violation 
of constraints $g$ is selected. In step 13, if $v_{i, j, t+1}$ does not satisfy the bounds defined by $Y$, then it is projected into the closest bound.

As in Algorithm 1, the steps marked with $\bullet$ change from one DE variant to the other. In Steps 8 and 10, DE/rand/1/bin randomly selects $r_{3} \in\{1, \ldots, N l / 2\}$ for $i \leq N l / 2$ and $r_{3}$ $\in\{N l / 2+1, \ldots, N l\}$ for $i>N l / 2$. The $D E /$ best/l/bin variant defines $y_{\text {best }}=y^{\prime}$ for $i \leq N l / 2$ and $y_{\text {best }}=y^{\prime \prime}$ for $i>N l / 2 ; y_{\text {best }}$ is used in Step 13 to replace $y_{r 3}$.

The acceptance criterion in step 18 (ACCEPT_LL) determines whether the new individual $v_{i, t+1}$ is accepted or not to replace $y_{i, t}$ in the next population. The acceptance criterion firstly observes whether the solutions $\left(x, v_{i, t+1}\right)$ and $\left(x, y_{i, t}\right)$ satisfy the lower level constraints $g(x, y) \leq 0$ ( $g$-feasibility), privileging the feasible solution if one of them is infeasible. If both are $g$-feasible, then it is checked whether they are nondominated w.r.t. to the current set of solutions Eff. If one of the solutions $v_{i, t+1}$ or $y_{i, t}$ is nondominated (i.e., it belongs to Eff) and the other is dominated, the nondominated solution is selected. If both solutions have the same status, the selection is based upon the upper level objective function value: for $i \leq N l / 2$ (sub-population oriented to the Optimistic frontier) the individual with lowest $F$ is selected; for $i>N l / 2$ (sub-population oriented to the Pessimistic frontier) the individual with highest $F$ is selected. It is worthwhile to note that, in an initial version of the algorithm, we did not use the set Eff in the acceptance criterion of $v_{i, t+1}$. The algorithm only compared the two candidate solutions, $v_{i, t+1}$ and

$y_{i, t}$, checking whether one dominated the other or both were nondominated w.r.t. to each other. However, the algorithm revealed a very poor convergence of the population to nondominated solutions, which was overcome with the current strategy.

\section{Computational Experiment}

The SVBLDE algorithm has been compared with the PSO algorithm in [1], which was extended to compute also the rewarding solution as this algorithm had been originally designed to determine the other three extreme solutions. Below we shortly designate the optimistic, pessimistic, deceiving and rewarding solutions by sol.o, sol.p, sol.d and sol.r, respectively (with $F^{o}, F^{p}, F^{d}$ and $F^{r}$ being the respective upper level objective values).

To test and compare the algorithms we have considered two sets of problems. The first set includes 4 problems - Prob.1 to Prob. 4 - whose formulations and sol.o, sol.p and sol.d are presented in [1]; these problems were adapted from the MOBL problems in [12] by considering only one upper level objective function. All the problems have one upper level variable and two lower level objective functions. Below we briefly describe these problems by indicating the number of lower level variables $\left(n_{2}\right)$ and showing the values of $F^{o}, F^{p}, F^{d}$ and $F^{r}$.

Prob. $1-n_{2}=2$; sol.o $\neq$ sol. $d \neq$ sol. $p$ with $F^{o}=0.5, F^{d}=1.25, F^{p}=1 ;$ sol. $r=$ sol.p, so $F^{r}=1$.

Prob. 2 - generalization of Prob. 1 with $n_{2}=k$. We consider $k=14$. The extreme solutions have the same characteristics as in Prob.1 and the same upper/lower level objective values. 
Prob. 3 and Prob. 4 have $n_{2}=2$ and differ from each other in the upper level objective function. They include an upper level constraint $G$ depending on lower level variables, which increases their difficulty. Prob.3: sol.o $=$ sol. $r$ with $F=-2$ and sol.d $=$ sol.p with $F=-1$. Prob.4: this problem admits alternative pessimistic solutions (all with $F^{p}=0$ ) but with different outcomes for the corresponding rewarding solution (with $F=-\alpha$, $0 \leq \alpha \leq 1$ ). The best rewarding solution corresponds to sol. $p=$ sol.d, $F^{p}=F^{d}=0$, being the rewarding solution sol. $r=$ sol.o with $F^{r}=F^{o}=-1$.

The second group of test problems are the MOBL problems DS1 to DS5 in [13], originally proposed in [14]. We consider only $F_{1}$ for the upper level objective function. This is a set of scalable problems with a variety of complex features to the algorithms. Problems DS1-DS3 have $k$ upper level and $k$ lower level variables - we consider $k=5$. Problems DS4 and DS5 have one upper level variable and $k+l$ lower level variables we consider $k=3$ and $l=2$. All the other parameters were set as in [13]. The corresponding values of $F^{o}, F^{p}, F^{d}$ and $F^{r}$ are presented in Table 1.

We have considered the following parameters for both algorithms, which were tuned through experimentation: $N u-N_{l}-T u-T_{l}$ equal to 20-60-50-100 for the first set of problems except Prob.2; 20-100-50-100 for Prob.2, DS4 and DS5, which also have one upper level variable but more than 2 lower level variables; 100-100-100-100 for DS1 to $D S 3$, which have a higher number of upper level variables. Specific parameters of the PSO algorithm were set as in [1]. We performed 30 independent runs of each algorithm in each problem.

Concerning the DE variants of the SVBLDE algorithm, we observed that the results of $D E / \mathrm{rand} / \mathrm{l} / \mathrm{bin}$ were not statistically different from the results of $\mathrm{DE} / \mathrm{best} / \mathrm{l} / \mathrm{bin}$ in about half of the cases; however, $D E /$ rand/1/bin provided very poor results in a few other cases. Therefore, and due to space reasons, we omit herein the results of that variant. Table 1 presents the median and the interquartile range IQR of the $F$ values obtained for the four extreme solutions over the 30 runs using the variant $\mathrm{DE} / \mathrm{best} / \mathrm{l} / \mathrm{bin}$ of SVBLDE and the PSO algorithm. We also include the exact values of $F$ (obtained analytically), which are very useful to assess the quality of the results obtained. The non-parametric Mann-Whitney test has been applied to assess whether the differences of the $F$ values obtained with the two algorithms are statistically significant, considering a confidence level of $95 \%$. The best result for each solution is highlighted in bold if the difference is statistically significant (' + ' in the last column).

Table 1. Median and interquartile range of $F$ in 30 independent runs for each algorithm.

\begin{tabular}{cl|cc|cc|c|c}
\hline \multirow{2}{*}{ Prob.1 } & \multicolumn{2}{|c|}{ SVBLDE } & \multicolumn{2}{c|}{ PSO algorithm } & \multicolumn{2}{c}{ M-W } \\
& & Median $F$ & IQR $F$ & Median $F$ & IQR $F$ & Exact $\boldsymbol{F}$ & test \\
\hline \multirow{3}{*}{ Prob.2 } & Sol.o & $\mathbf{0 . 4 9 7 3 8 4}$ & 0.000708 & 0.496248 & 0.001489 & $\mathbf{0 . 5}$ & + \\
& Sol.p & 0.993762 & $7.04 \mathrm{E}-05$ & 0.993742 & $9.36 \mathrm{E}-05$ & $\mathbf{1}$ & - \\
& Sol.d & 1.246284 & 0.01238 & 1.246038 & 0.016857 & $\mathbf{1 . 2 5}$ & - \\
& Sol.r & 0.993713 & 0.004252 & 0.988769 & 0.021896 & $\mathbf{1}$ & - \\
\hline \multirow{3}{*}{ Prob.3 3} & Sol.o & $\mathbf{0 . 4 8 7 3 9 7}$ & 0.002296 & 0.407539 & 0.026634 & $\mathbf{0 . 5}$ & + \\
& Sol.p & $\mathbf{0 . 9 9 9 2 2 0}$ & 0.011627 & 0.991885 & 0.000691 & $\mathbf{1}$ & + \\
& Sol.d & $\mathbf{1 . 2 5 0 1 3 8}$ & 0.035337 & 1.202307 & 0.069874 & $\mathbf{1 . 2 5}$ & + \\
& Sol.r & 0.908306 & 0.057767 & $\mathbf{0 . 9 8 6 0 3}$ & 0.007036 & $\mathbf{1}$ & + \\
\hline & Sol.o & -2 & 0.006734 & -1.99995 & $4.01 \mathrm{E}-05$ & $\mathbf{- 2}$ & - \\
& Sol.p & -0.99985 & 0.001206 & -0.99984 & 0.000215 & $\mathbf{- 1}$ & - \\
& Sol.d & -1.00214 & 0.003443 & -1.00296 & 0.001645 & $\mathbf{- 2}$ & - \\
\hline
\end{tabular}




\begin{tabular}{|c|c|c|c|c|c|c|c|}
\hline \multirow{4}{*}{ Prob.4 } & Sol.o & -0.99694 & 0.007655 & -0.99995 & $5.12 \mathrm{E}-05$ & -1 & - \\
\hline & Sol.p & -0.00356 & 0.001833 & -0.00606 & 0.000695 & 0 & + \\
\hline & Sol.d & -0.00334 & 0.001618 & -0.00391 & 0.001138 & 0 & - \\
\hline & Sol.r & -0.96020 & 0.092859 & -0.89689 & 0.111896 & -1 & + \\
\hline \multirow{4}{*}{$D S 1$} & Sol.o & $2,51 \mathrm{E}-05$ & $3,1 \mathrm{E}-05$ & $5,61 \mathrm{E}-05$ & $3,11 \mathrm{E}-05$ & $\mathbf{0}$ & + \\
\hline & Sol.p & 0,07746 & 0,056167 & 0,099769 & 0,000128 & 0.1 & + \\
\hline & Sol.d & 0,092602 & 0,061951 & 0,099981 & 0,000168 & 0.1 & + \\
\hline & Sol.r & $3,23 E-05$ & 0,000107 & 0,000179 & 0,000193 & $\mathbf{0}$ & + \\
\hline \multirow{4}{*}{$D S 2$} & Sol.o & $-0,25977$ & 0,013058 & $-0,34826$ & 0,035846 & -0.238773 & + \\
\hline & Sol.p & $-0,23876$ & $8,07 \mathrm{E}-06$ & $-0,23877$ & $2,68 \mathrm{E}-06$ & -0.238773 & + \\
\hline & Sol.d & $-0,23873$ & 0,000247 & $-0,23877$ & $9,51 \mathrm{E}-07$ & -0.238773 & + \\
\hline & Sol.r & $-0,23876$ & $1,36 \mathrm{E}-05$ & $-0,23878$ & 0,091723 & -0.238773 & + \\
\hline \multirow{4}{*}{$D S 3$} & Sol.o & 1.85E-07 & $2.64 \mathrm{E}-07$ & $5.34 \mathrm{E}-05$ & $9.29 \mathrm{E}-05$ & $\mathbf{0}$ & + \\
\hline & Sol.p & $1.84 \mathrm{E}-07$ & $1.03 \mathrm{E}-07$ & 0.200086 & $1.65 \mathrm{E}-05$ & 0.2 & + \\
\hline & Sol.d & $1.86 \mathrm{E}-07$ & $1.83 \mathrm{E}-07$ & 0.200299 & 0.000192 & 0.2 & + \\
\hline & Sol.r & 1.99E-07 & $2.65 \mathrm{E}-07$ & 0.001461 & 0.004658 & $\mathbf{0}$ & + \\
\hline \multirow{4}{*}{$D S 4$} & Sol.o & $\mathbf{0}$ & 0 & 0 & 0.845635 & $\mathbf{0}$ & + \\
\hline & Sol.p & 102 & 0 & 102 & 0 & 102 & - \\
\hline & Sol.d & 204 & 0 & 204 & 100.5451 & 204 & + \\
\hline & Sol.r & 1.000245 & 0.000347 & 2.388914 & 0.519674 & 1 & + \\
\hline \multirow{4}{*}{ DS5 } & Sol.o & 0.760132 & 0.000174 & 2.01667 & 0.021934 & 0.76 & + \\
\hline & Sol.p & 102 & 0 & 102 & 0 & 102 & - \\
\hline & Sol.d & 188.9164 & 27.86941 & 102 & 0 & 167.3 & + \\
\hline & Sol.r & 1.000139 & 0.000134 & 2.268107 & 0.36943 & 1 & + \\
\hline
\end{tabular}

It is noteworthy that there are several difficulties in evaluating results to SVBLP. These difficulties can easily lead to pitfalls in the interpretation of results, which may be very difficult to avoid in general problems for which the exact solutions are not known. We draw attention to some of these pitfalls:

- Only efficient (Pareto optimal) solutions to the lower level problem are feasible to the SVBLP. Therefore, an algorithm may yield apparently better solutions (for any of the four extreme solutions), i.e. with lower $F$ values, but the solutions are invalid because they are not efficient to the lower level problem.

- Even if only efficient (Pareto optimal) solutions to the lower level problem are obtained, other difficulties arise in assessing the pessimistic and deceiving solutions. Solutions with lower $F$ values (i.e., which seem to be better) may be false because they are not in the Pessimistic frontier, i.e., they are not the worst for the leader for that setting of $x$. We can observe this situation in Table 1 for several sol.d and sol.p (e.g., Prob.2, Prob.4, DS1, DS3 or DS5).

From Table 1, we observe that SVBLDE outperformed the PSO algorithm in 17 out of the 36 cases (4 extreme solutions to 9 problems) while the PSO algorithm outperformed SVBLDE in 9 cases (the differences in the other 10 cases were not statistically significant). Therefore, SVBLDE seems to perform slightly better than the PSO algorithm. We can also observe that SVBLDE is very effective in approximating the optimistic solution, being always better or equal to the PSO algorithm, but SVBLDE reveals more difficulty in attaining the real pessimistic and deceiving solutions in several cases. 


\section{Conclusions}

We presented a new DE algorithm to compute the optimistic/deceiving and pessimistic/rewarding solutions to the SVBLP. These four extreme solutions capture the optimistic vs. pessimistic leader's attitude and possible follower's reactions more or less favorable to the leader. The DE approach seems to perform slightly better than the PSObased approach, but the results do not evidence a clear performance advantage of the SVBLPDE algorithm with respect to PSO. The experiments unveiled some pitfalls associated with the interpretation of results and assessment of the algorithm performance in SVBLP. These pitfalls could be avoided because we were able to determine analytically the exact solutions to the problems tested. Research is underway on techniques to mitigate these pitfalls in general problems, which are nevertheless intrinsic to this kind of problems and cannot be entirely avoided.

Acknowledgment. This work was supported by projects UID/MULTI/00308/2013 and SAICTPAC/0004/2015-POCI-01-0145-FEDER-016434.

\section{$7 \quad$ References}

[1] Alves MJ, Antunes CH, Carrasqueira P. A PSO Approach to Semivectorial Bilevel Programming: Pessimistic, Optimistic and Deceiving Solutions. Proc. Genet. Evol. Comput. Conf. (GECCO 2015), 2015, p. 599-606.

[2] Bonnel H. Optimality conditions for the semivectorial bilevel optimization problem. Pacific J Optim 2006;2:447-68.

[3] Bonnel H, Morgan J. Semivectorial bilevel optimization problem: penalty approach. J Optim Theory Appl 2006;131:365-82.

[4] Ankhili Z, Mansouri A. An exact penalty on bilevel programs with linear vector optimization lower level. Eur J Oper Res 2009;197:36-41.

[5] Zheng Y, Wan Z. A solution method for semivectorial bilevel programming problem via penalty method. J Appl Math Comput 2011;37:207-19.

[6] Ren A, Wang Y. A novel penalty function method for semivectorial bilevel programming problem. Appl Math Model 2016;40:135-49.

[7] Calvete H, Galé C. On linear bilevel problems with multiple objectives at the lower level. Omega 2011;39:33-40.

[8] Liu B, Wan Z, Chen J, Wang G. Optimality conditions for pessimistic semivectorial bilevel programming problems. J Inequalities Appl 2014;2014:41.

[9] Lv Y, Chen J. A Discretization Iteration Approach for Solving a Class of Semivectorial Bilevel Programming Problem. J Nonlinear Sci Appl 2016;9:2888-99.

[10] Alves, M. J., Antunes CH. An illustration of different concepts of solutions in semivectorial bilevel programming. 2016 IEEE Symp. Comput. Intell. (SSCI); 2016.

[11] Mezura-Montes E, Velázquez-Reyes J, Coello Coello CA. A comparative study of differential evolution variants for global optimization. Proc. 8th Annu. Conf. Genet. Evol. Comput., 2006, p. 485-92.

[12] Deb K, Sinha A. Solving bilevel multi-objective optimization problems using evolutionary algorithms. Proc. EMO 2009, LNCS 5467, Springer; 2009, p. 110-24.

[13] Sinha A, Malo P, Deb K. Approximated set-valued mapping approach for handling multiobjective bilevel problems. Comput Oper Res 2017;77:194-209.

[14] Deb K, Sinha A. Constructing test problems for bilevel evolutionary multi-objective optimization. 2009 IEEE Congr. Evol. Comput., 2009, p. 1153-60. 